\title{
Predictors for the development of cholangiocarcinoma and hepatocellular carcinoma: in search for the most accurate biomarker
}

\author{
Alexandros A. Tzovaras ${ }^{1}$, Konstantinos Kamposioras ${ }^{2}$, Alexandros Ardavanis ${ }^{1}$
}

${ }_{11}^{1}$ st Department of Medical Oncology, Aghios Savvas Anticancer Hospital, Athens, Greece

2Department of Medical Oncology, Leeds Teaching Hospital, NHS Trust, Leeds, United Kingdom

Submitted: 17 December 2011

Accepted: 19 December 2011

Arch Med Sci 2011; 7, 6: 925-927

DOI: 10.5114/AOMS.2011.26600

Copyright ( 2011 Termedia \& Banach

\section{Introduction}

Daduang et al. in this issue of Archives of Medical Science evaluate a disintegrin and metalloprotease (ADAM) 12 level as a distinguishing biomarker between hepatocellular carcinoma (HCC) and cholangiocarcinoma (CC) [1]. Mean serum levels of ADAM12 in their study were higher in HCC compared with both CC and healthy controls ( $p<0.001$ for both combinations). Regarding specificity and positive likelihood ratio (PLR), ADAM12 had better specificity and PLR for HCC compared with CC (specificity: $77.4 \%$ vs. $64.5 \%$, respectively; PLR: 3.51 vs. 1.25 , respectively). Furthermore, serum levels of ADAM12 in HCC correlated with the overall survival $(p=0.02)$, indicating that high serum level of ADAM12 are associated with a significantly poorer prognosis. The conclusion reached was that ADAM12 may be used as an adjunct biomarker for the differential diagnosis between HCC and CC.

Some additional comments may be of interest.

\section{Differential diagnosis between hepatocellular carcinoma and cholangiocarcinoma: where are we now?}

Cholangiocarcinoma accounts for about $3 \%$ of all gastrointestinal tumors and about $15 \%$ of all primary liver cancers worldwide [2]. The lack of early detection and the limited therapeutic options available are major problems in the management of CC [2]. Whether intrahepatic immune cells play a role in the pathogenesis or treatment of hepatic cancers [3] is also another topic requiring further investigation.

The differential diagnosis between HCC and CC is not easily established $[4,5]$. There are many diversities and pitfalls in the role of genetic factors in the development of HCC [5], but not CC. Cholangiocarcinoma is more common in females and in more elderly patients compared with HCC. Furthermore, CC has a lower proportion of asymptomatic tumors, lower serum $\alpha$-fetoprotein, higher serum carcinoembryonic antigen, carbohydrate antigen (CA) 19-9 and alkaline phosphatase levels compared with HCC. In CC, there is also a lower incidence of hepatitis history, associated cirrhosis and serum hepatitis B surface antigen, a lower proportion of small/wellencapsulated tumors and tumor emboli in the portal vein, a higher pro-
Corresponding author: Alexandros A. Tzovaras MD 36 Aghias Paraskevis St. Kifissia 14561

Athens, Greece

E-mail: alextzo74@yahoo.gr 
portion of single tumor, perihilar lymph node involvement and poor differentiation and less frequency of limited resection compared with HCC (for all associations $p<0.0001)[4,5]$.

Although CA19-9 is the most available marker for the detection of pancreatic cancers, CC and other pancreatic malignancies, it may not be an adequate marker for the differential diagnosis between the 2 types of tumor [6]. The reason for this is that the sensitivity and specificity of CA19-9 is often suboptimal in clinical practice [6]. Furthermore, many patients with elevated CA 19-9 levels do not have CC, but instead more benign conditions such as primary sclerosing cholangiitis [7].

A variety of other markers have been proposed to have a diagnostic or prognostic value for the 2 tumor types. such as $\alpha v \beta 6$ integrin [8]. NAD(P) $\mathrm{H}$ :quinone oxidoreductase-1 (NQO1) [9], MUC1 [10] and thyroid transcription factor (TTF)-1 [11]. The $\alpha v \beta 6$ integrin is strongly expressed in human CC, but not in HCC and can therefore be considered as a specific immunohistochemical marker in the differential diagnosis of primary liver tumors [8]. A considerable drawback of all the above-mentioned markers [8-11] is that they are tissue (not blood) marker.

A study from China analyzed 5 different serum tumor markers ( $\alpha$-fetoprotein, carcinoembryonic antigen, CA19-9, CA242 and CA50) for their ability to establish the differential diagnosis between CC and HCC [12]. Serum levels and the positive rate of $\alpha$-fetoprotein, CA19-9 and CA242 were different between the CC and HCC. Additionally, the combination of AFP (-) and CA242 (+) afforded a high specificity of $94.3 \%$ and the highest accuracy (that is $78.5 \%$ ) [12].

In their small study ( $n=21$ patients), Sriwanitchrak et al. attempted to identify novel serum markers which can substitute CA19-9, or can improve the diagnostic accuracy of CA19-9 when measured together with this marker [2]. Based on the results of binary logistic regression analysis of 3 serum biomarkers (CA19-9, antitrypsin-1 and $\alpha$-fetoprotein), serum levels of CA19-9 together with antitrypsin-1 were the minimum requirement to obtain prediction accuracy of greater than $80 \%$ in a battery test for diagnosis of CCA. However, in order to obtain high predictability, an addition of at least 1 of the 3 liver function enzymes (alkaline phosphatase, aspartase transaminase or alanine trasaminase) was required. The conclusion reached was that serum biomarkers may be a useful diagnostic or prognostic monitoring tool for CC, but further evaluation in larger trials is required [2].

The lack of a single reliable predictor for the detection and diagnosis of hepatic tumors has lead to the development of the so-called Fudan score [13]. The Fudan score is a combination of various independent predictors, namely serum alkaline phosphatase level, CA 19-9 level, tumor boundary type, tumor size and number of intrahepatic tumors. A Fudan score may provide a relatively accurate prognostic prediction of CC regardless of resection status [13].

As mentioned by Daduang et al. [1], serum levels of ADAM12 in HCC are inversely associated with the overall survival $(p=0.02)$, indicating that high serum level of ADAM12 are associated with a significantly poorer prognosis. Future studies should evaluate whether the addition of ADAM12 in the above mentioned parameters comprising the Fudan score could provide additional predictive value in the detection and long-term prognosis of these 2 types of tumor. Furthermore, future studies should evaluate whether serum biomarkers may be a useful diagnostic or prognostic monitoring tool for CC.

\section{References}

1. Daduang J, Limpaiboon T, Daduang S. Biomarker to distinguish hepatocellular carcinoma from cholangiocarcinoma by serum a disintegrin and metalloprotease 12 . Arch Med Sci 2011; 7: 1013-6.

2. Sriwanitchrak P, Viyanant V, Chaijaroenkul W, et al. Proteomic analysis and evaluation of biomarkers for detection of cholangiocarcinoma. Asian Pac J Cancer Prev 2011; 12: 1503-10.

3. Bassiouny AE, El-Hassan SA, Moussa M, et al. Down-regulation of intrahepatic CD16+ and CD56+ immune cells in chronic Hepatitis C virus infection and HCV-related hepatocellular carcinoma. Arch Med Sci 2009; 5: 321-8.

4. Zhou XD, Tang ZY, Fan J, et al. Intrahepatic cholangiocarcinoma: report of 272 patients compared with5.829 patients with hepatocellular carcinoma. J Cancer Res Clin Oncol 2009; 135: 1073-80.

5. El-Din HG, Ghafar NA, Saad NE, Aziz M, Rasheed D, Hassan EM. Relationship between codon 249 mutation in exon 7 of p53 gene and diagnosis of hepatocellular carcinoma. Arch Med Sci 2010; 6: 348-55.

6. Singh S, Tang SJ, Sreenarasimhaiah J, Lara LF, Siddiqui A. The clinical utility and limitations of serum carbohydrate antigen (CA19-9) as a diagnostic tool for pancreatic cancer and cholangiocarcinoma. Dig Dis Sci 2011; 56: 2491-6.

7. Sinakos E, Saenger AK, Keach J, Kim WR, Lindor KD. Many patients with primary sclerosing cholangitis and increased serum levels of carbohydrate antigen 19-9 do not have cholangiocarcinoma. Clin Gastroenterol Hepatol 2011; 9: 434-9.e1.

8. Patsenker E, Wilkens L, Banz V, et al. The alphavbeta6 integrin is a highly specific immunohistochemical marker for cholangiocarcinoma. J Hepatol 2010; 52: 362-9.

9. Wakai T, Shirai Y, Sakata J, et al. Prognostic significance of NQO1 expression in intrahepatic cholangiocarcinoma. Int J Clin Path 2011; 4: 363-70.

10. Yuan SF, Li KZ, Wang L, et al. Expression of MUC1 and its significance in hepatocellular and cholangiocarcinoma tissue. World J Gastroenterol 2005; 11: 4661-6.

11. Lei JY, Bourne PA, di Sant'Agnese PA, Huang J. Cytoplasmic staining of TTF-1 in the differential diagnosis of hepatocellular carcinoma vs cholangiocarcinoma and metasta- 
tic carcinoma of the liver. Am J Clin Pathol 2006; 125:

519-25.

12. Tao LY, Cai L, He XD, Liu W, Ou O. Comparison of serum tumor markers for intrahepatic cholangiocarcinoma and hepatocellular carcinoma. Am Surg 2010; 76: 1210-3.

13. Jiang W, Zeng ZC, Tang ZY, et al. A prognostic scoring system based on clinical features of intrahepatic cholangiocarcinoma: the Fudan score. Ann Oncol 2011; 22: 1644-52. 